\title{
Mediación de la información en el ámbito del gobierno corporativo
}

Information mediation in the corporate governance environment

\author{
Elaine Cristina LoPes (1) y Marta Lígia Pomim Valentim (2) \\ UNESP - Universidade Estadual Paulista Júlio de Mesquita Filho, Faculdade de Filosofia e Ciências - \\ Campus de Marília, Cidade Universitária - Marília, SP - Brasil, Caixa Postal 181, \\ elainelopes@marilia.unesp.br. (2) valentim@marilia.unesp.brResumen
}

\begin{abstract}
Resumen
Este artículo presenta algunos modelos de gestión estratégica de la información y de gestión del conocimiento en empresas con capital abierto, con un nivel de gobierno corporativo. Estos conceptos se basan en la divulgación transparente y coherente de informaciones relevantes para los negocios de la empresa. Se consideran, además, los padtones de mediación de esas informaciones entre las empresas y los accionistas minoritarios y los efectos de esta mediación en la toma de decisiones que realizan los inversores.
\end{abstract}

Palabras clave: Mediación de la información. Mercado de capitales. Gestión de la información. Flujos de información. Gobierno corporativo. Toma de decisiones.

\section{Introducción}

A la luz de los cambios tecnológicos y comerciales de los últimos decenios, se vivencia actualmente un escenario de creciente reconocimiento del poder de la información y del conocimiento. Independientemente del sector, las organizaciones se están preparando para el desarrollo basado en la creación de estrategias apoyadas en informaciones de distinta naturaleza, que a su vez propician una base para la creación del conocimiento, cuyo contenido es esencial para alcanzar los mercados externos.

Es evidente que el impacto puede ser generado a partir de ciertas informaciones, principalmente cuando llegan al ambiente externo a la empresa. Sean impactos positivos o negativos, ellos ocurren y, por lo tanto, es necesaria la correcta gestión de dichas informaciones. Como resultado de ello, es esencial considerar cómo esas informaciones llegan al público externo; o sea, llevar a cabo una correcta mediación de la información es esencial para cualquier empresa de capital abierto.

\begin{abstract}
This paper presents some models of strategic information management and knowledge management in companies with open capital, with a level of corporate governance. These concepts are based on transparency and consistent disclosure of relevant information on the company's businesses. It also considers the patterns of mediation of such information between the companies and minority shareholders, and the impacts of this mediation on investors' decision-making.
\end{abstract}

Keywords: Information Mediation. Capital Market. Information Management. Information Flows. Corporate Governance. Decision-Making Process.

Las empresas con acciones negociadas en las bolsas de valores tienen características específicas en lo que se refiere a la gestión de la información y del conocimiento. Por eso, en la última década surgió la necesidad de crear mecanismos que aproximase a los inversionistas a través de la imagen institucional. El gobierno corporativo nació de esa necesidad y es un concepto en fuerte desarrollo, por establecer primordialmente la equidad en las relaciones entre accionistas minoritarios y mayoritarios a través de la gestión de la información. En este contexto de urgencia por las informaciones, y por creación del conocimiento para la toma de decisiones, es fundamental el análisis del mediador y de los medios de comunicación usados en la diseminación de la informaciones.

\section{Gestión de la información y del conocimiento en el contexto empresarial}

La gestión estratégica de la información es de una importancia creciente en lo que se refiere al desarrollo empresarial mundial. El uso de las nuevas tecnologías de información y comunica- 
ción y la velocidad con que los datos son convergidos en informaciones es una realidad en el medio organizacional, una vez que el impacto de determinadas informaciones cuando llegan al mercado debe hacer parte del esfuerzo organizacional para la elaboración de las estrategias empresariales. Davenport y Prusak (1998) comentan que, en los últimos decenios, los ejecutivos de las empresas estaban satisfechos con la mera distribución de informaciones cuantitativas; no obstante, en el mundo actual existe la necesidad de construir conocimientos, reflexionar sobre un determinado problema y principalmente, tomar decisiones de manera asertiva.

En este contexto de complejidad -donde las organizaciones son constantemente impulsadas a dinamizar sus procesos informacionales, motivadas por la concurrencia, por las turbulencias económicas, las demandas sociales y comerciales- es donde el concepto de gestión de la información y del conocimiento se desarrolla. Estos conceptos surgen de la necesidad de llevar a cabo la gestión de la información, a fin de generar conocimiento, cuya dinámica alimenta una a otra, y son posibles por la capacidad individual y colectiva de las personas y de los procesos utilizados para compartir esos conocimientos (Valentim; Gelinski, 2006, p. 120).

La gestión del conocimiento en el contexto de la inteligencia competitiva organizacional puede ser entendida como un sistema que abarca todos los flujos y procesos relacionados con el fomento de la generación, compartimiento, sistematización y diseminación del conocimiento en el ambiente corporativo, con el fin de subvencionar el desarrollo de actividades/tareas y el proceso de toma de decisiones y, por consiguiente, para obtener ventaja competitiva.

Cada vez más, los empresarios y ejecutivos entienden el importante papel de la información y del conocimiento, porque se constituyen en mecanismos para lograr el éxito de una estrategia organizacional. La información es parte de cualquier actividad que una empresa realiza, desde el desarrollo de una idea, la elaboración de un proyecto, hasta la transformación de una idea en un producto o servicio al consumidor final. Sin embargo, utilizar las informaciones generadas en la rutina de la empresa, y utilizar las informaciones generadas externamente a la empresa como una forma de crear conocimiento, puede convertirse en un importante diferencial. Para Choo (2006, p. 28), el uso estratégico de la información consiste en que se crea, organiza y procesa la información para generar nuevos conocimientos que permitan a la empresa desarrollar nuevas competencias, nuevos productos y servicios, mejorar los existentes y perfeccionar los procesos organizacionales.
Ocurre que, en muchos casos, el conocimiento necesario para mantener la empresa competitiva en el mercado y aumentar su desempeño ya se encuentra dentro de la propia empresa. Sin embargo, no se lo interpreta y, por tanto, no es exteriorizado. En ese sentido, es esencial que la empresa sea capaz de comprender la importancia de la información y del conocimiento y se vea como una generadora de conocimientos a fin de crear mecanismos para el aprovechamiento de esa competencia.

Choo (2006, p. 231) explica que las organizaciones pueden verse como los repositorios de las capacidades que se derivan del hecho de que el conocimiento de los individuos y grupos de la empresa han ganado forma y coherencia, a través de las relaciones, procesos y herramientas que definen la empresa a lo largo del tiempo.

Esto refuerza la idea de que de nada sirve la generación del conocimiento, sin una gestión eficaz centrada en el conocimiento generado. El conocimiento individual y colectivo es el resultado de la cultura de la empresa, la formación de las personas y del contexto en que la empresa está insertada. Cabe a la empresa la elaboración de instrumentos para identificar las informaciones fundamentales para el proceso de creación del conocimiento. Todo esto marca fuertemente el paso de la Era Industrial a la Era de la Información, toda vez que, más que un soporte, la información y el conocimiento pueden ser considerados activos de la empresa. Chiavenato (2002, p. 478) destaca que en la Era de la Información, el capital financiero cede el lugar al capital intelectual, y convierte el conocimiento en la nueva riqueza, el recurso más valioso e importante.

\section{Capitalización empresarial vía apertura de capital}

Una de las más importantes decisiones que se adoptan en una empresa, se refiere a la hora y la manera en que realizará las inversiones de corto, mediano y largo plazo. La decisión de inversión de la empresa, especialmente en un entorno de incertidumbre, se refiere al tipo de inversión y los beneficios previstos, considerando que los resultados sean mayores que los costos involucrados para su implantación. Para Dutra $(2003$, p. 18) las nuevas tecnologías, la implantación de sistemas complejos de producción y, consecuentemente, el crecimiento de la empresa requieren un mayor control y seguridad en las aplicaciones de capital y en el mantenimiento de los sistemas de producción con la 
presentación de resultados positivos en las operaciones realizadas.

Existen diversas formas de capitalización de la empresa, incluyendo la apertura de capital, que se trata de la venta de acciones de la empresa a un público diverso, llamado comúnmente el inversionista. Por lo tanto, hay dos intereses distintos, por un lado la empresa que pretende aumentar los recursos para las inversiones y por otro lado el ahorrador, persona física o jurídica, que tiene como objetivo proporcionar el máximo de inversión de su capital.

Las operaciones de compra y venta de acciones son realizadas, fiscalizadas y normatizadas en el ámbito del mercado de capitales, que, a su vez, es parte del sistema financiero brasileño. La comercialización de las acciones de las empresas se lleva a cabo en las Bolsas de Valores, que se definen, como explica Fortuna (2005), como asociaciones civiles sin fines de lucro, creadas por el corretaje de valores para proporcionar la infraestructura para el mercado de acciones. Las instituciones encargadas de la intermediación, o de la operación de la compra y venta de las acciones, son las sociedades de corretaje, que operan dentro de las bolsas de valores y hacen público el lanzamiento de acciones de las empresas, entre otras tareas relevantes para el mercado de capitales.

Sin embargo, aunque la mayor razón que lleva a una empresa a abrir su capital sea la recaudación de fondos para la inversión, una empresa de capital tiene un diferencial competitivo en relación a los competidores y establece una relación de mayor confianza con sus socios comerciales. Todo esto se deriva de la exigencia del público y del propio sistema financiero de que haya transparencia y fiabilidad de las informaciones y, especialmente, de la rendición de cuentas, un factor que permite a la empresa un mayor poder de negociación con sus proveedores y consumidores, además de proporcionarle una mayor visibilidad frente al mercado y la prensa.

La alternativa de abrir el capital en detrimento de la recaudación de fondos a través de préstamos o de la inyección de fondos de los accionistas está intrínsecamente vinculada al desarrollo económico del país. El crecimiento de las empresas genera un aumento en la producción de bienes y servicios, nuevos empleos, aumenta el consumo de la población y, por tanto, genera más ingresos procedentes de impuestos al gobierno. Para el inversor que adquiere acciones de una empresa, ocurre la formación de ahorro a medio y largo plazo, costumbre que no se cultiva en Brasil.

\section{Gobierno corporativo y transparencia en la divulgación de informaciones}

\subsection{Principios fundamentales}

El gobierno corporativo puede entenderse como un conjunto de prácticas orientadas a aproximar los intereses de los accionistas mayoritarios y de los inversores, representados por los accionistas minoritarios. Estos conceptos han nacido de la busca de un modelo para definir el ámbito de los valores, principios, normas y procedimientos que podrían establecer los conceptos de propiedad, derechos y obligaciones de ambas partes.

La labor de las organizaciones multilaterales como la Organización para Cooperación y Desarrollo Económico (OCDE) y el Instituto Brasileiro de Governança Corporativa (IBGC) se orientan por los conceptos de buen gobierno corporativo, buscando una integración de procesos relacionados con la calidad de las informaciones que orientan los inversores en su toma de decisiones. Para el IBGC (2008) las buenas prácticas de gobierno corporativo tienen la finalidad de aumentar el valor de la empresa, facilitar su acceso al capital y contribuir a su perennidad.

El propósito fundamental del gobierno corporativo es la atención para mejorar el diálogo entre los administradores y el mercado para asegurar que cada grupo tenga igual nivel de conocimientos sobre las informaciones relevantes acerca de la empresa, desde el desempeño de los negocios hasta los resultados financieros. En este sentido, Williamson (1996, apud Andrade, 2004, p. 24) señala que "el gobierno corporativo trata de la justicia, de la transparencia y de la responsabilidad de las empresas cuanto a las cuestiones que rodean a los intereses del negocio y de la sociedad en su conjunto".

Como principios fundadores, destacan los conceptos considerados como los valores que sostienen el buen gobierno corporativo. Estos valores son la transparencia, la equidad y la rendición de cuentas, también conocidos como la triada del gobierno corporativo. Para el IBGC (2008), son fundamentales la transparencia, la buena comunicación interna y externa - sobre todo cuando es espontánea, libre y rápida, que resultan en un clima de confianza, tanto internamente como en las relaciones de la empresa con terceros-, así como la equidad —que se caracteriza por un tratamiento justo y equitativo de todos los grupos minoritarios- y, por último, la importancia de los agentes de la empresa, con respecto a la rendición de cuentas de las acciones para las que hayan sido elegidos, y 
que respondan plenamente de todos los actos que practicaren en el ejercicio de sus mandatos.

\subsection{Prácticas de gobierno corporativo en Brasil}

En Brasil, las prácticas de gobierno corporativo han ganado fuerza en los últimos cinco años, muy influidas por el hecho de que las características estructurales de las empresas brasileñas afectan a las relaciones entre los accionistas minoritarios y mayoritarios. La alta concentración de propiedad, con indicación de la detención de más del $80 \%$ por pocos accionistas, el predominio de las empresas familiares y, sobre todo, la desconsideración de los intereses de los accionistas minoritarios, motivó a los órganos internacionales y nacionales en la búsqueda de una aproximación de intereses.

En Brasil, el gobierno corporativo ha sido desarrollado a partir de los esfuerzos de diversos organismos como del Instituto Brasileiro de Governança Corporativa (IBGC), la Associação Brasileira de Companhias Abertas (ABRASCA), el Comitê de Orientação para Divulgação de Informações ao Mercado (CODIM), la Comissão de Valores Mobiliários (CVM), y la Bolsa de Valores de São Paulo (BOVESPA), entre otros. Estas prácticas están justificadas por la existencia de normas y reglamentos que tratan de orientar la conducta de las empresas, especialmente en la forma de difundir informaciones. En la Tabla I se presentarán algunos de estos esfuerzos, que representan un marco para el proceso de desarrollo de los conceptos del gobierno corporativo.

Los conflictos de interés y la necesidad de mejorar la imagen corporativa frente al mercado crearon la necesidad de establecer condiciones para el avanzo en la definición de padrones mundiales. Para Andrade (2004, p. 350) el gobierno corporativo en Brasil es influenciado por una amplia gama de fuerzas internas y externas que afectan a los valores, principios y modelos practicados.

\section{Mediación de la información y los parámetros del gobierno corporativo}

En las operaciones del mercado de capitales, las informaciones son fundamentales en la relación entre la empresa y los inversores. Esta afirmación se basa en el hecho de que cualquier información puede ser utilizada por los inversores en el momento de la decisión de compra o venta de una acción. Se pueden contemplar en los periódicos y telediarios las noticias relacionadas a las fluctuaciones de las Bolsas de Valores mundiales motivadas por informaciones divulgadas al mercado. Estas fluctuaciones se refieren a los precios de acciones de empresas, que suben y bajan de valor de acuerdo con las fuerzas de los compradores y vendedores, en la mayoría de los casos, motivados por informaciones pertinentes sobre las empresas, la economía, la política.

\begin{tabular}{|c|c|}
\hline Acciones & Objetivos \\
\hline $\begin{array}{l}\text { Recomendaciones de } \\
\text { la Comissão de } \\
\text { Valores Mobiliários } \\
\text { (CVM) }\end{array}$ & $\begin{array}{l}\text { Disposiciones sobre las normas } \\
\text { de conducta centradas en } \\
\text { cuestiones tales como } \\
\text { asambleas, estructura } \\
\text { accionarial, protección a los } \\
\text { accionistas minoritarios, } \\
\text { divulgación de informaciones, } \\
\text { etc. }\end{array}$ \\
\hline $\begin{array}{l}\text { Código Brasileño de } \\
\text { Mejores Prácticas de } \\
\text { Gobierno Corporativo } \\
\text { del Instituto Brasileiro } \\
\text { de Governança } \\
\text { Corporativa (IBGC) }\end{array}$ & $\begin{array}{l}\text { Proponer recomendaciones que } \\
\text { sirvan como parámetros para las } \\
\text { empresas que deseen seguir las } \\
\text { bases de las buenas prácticas } \\
\text { de gobierno corporativo en } \\
\text { Brasil. }\end{array}$ \\
\hline $\begin{array}{l}\text { Manual de Control y } \\
\text { Difusión de } \\
\text { Informaciones } \\
\text { Pertinentes de la } \\
\text { Associação Brasileira } \\
\text { de Companhias } \\
\text { Abertas (ABRASCA) } \\
\end{array}$ & $\begin{array}{l}\text { Instruir a las empresas con } \\
\text { capital abierto en el correcto } \\
\text { tratamiento de las informaciones } \\
\text { privilegiadas, con el objetivo } \\
\text { principal de garantizar la ética y } \\
\text { la discreción de esas } \\
\text { informaciones. }\end{array}$ \\
\hline $\begin{array}{l}\text { Niveles diferenciados } \\
\text { de Gobierno } \\
\text { Corporativo de la } \\
\text { Bolsa de Valores de } \\
\text { São Paulo } \\
\text { (BOVESPA) }\end{array}$ & $\begin{array}{l}\text { Seguimientos especiales de } \\
\text { listado que poseen objetivo de } \\
\text { establecer reglas propias con un } \\
\text { grado de compromiso con el } \\
\text { inversionista superior a la } \\
\text { legislación vigente. }\end{array}$ \\
\hline $\begin{array}{l}\text { Pronunciamientos de } \\
\text { Orientaciones del } \\
\text { Comitê de Orientação } \\
\text { para Divulgação de } \\
\text { Informações ao } \\
\text { Mercado (CODIM) }\end{array}$ & $\begin{array}{l}\text { Pronunciamientos que reflejan } \\
\text { temas sobre las mejores } \\
\text { prácticas de difusión, a fin de } \\
\text { establecer principios básicos de } \\
\text { divulgación. Entre los temas está } \\
\text { el uso de recursos tecnológicos } \\
\text { compatibles con las necesidades } \\
\text { de informaciones del mercado. }\end{array}$ \\
\hline
\end{tabular}

Tabla I. Acciones de incentivos para el buen gobierno corporativo.

Partiendo del principio de que muchas decisiones son tomadas por los inversores a la luz de las informaciones divulgadas, no se debe ignorar la mediación de la información, ya que esto es clave fundamental para el proceso de aproximación del poder de control de la empresa y los accionistas minoritarios. Por supuesto, las informaciones no se refieren solo al universo individual de las empresas; es decir, no se puede mirar sólo desde una perspectiva microeconómica, ya que las grandes crisis son de orden macroeconómico, como resultado de factores políticos y económicos. Sin embargo, en este caso, se consideran únicamente las informaciones de carácter específico de las empresas. 
Así pues, la mediación de la información se basará en lo que respecta a la necesidad de información. En este contexto, los inversores necesitan de un conjunto de información que utilizarán para componer su propio conocimiento, condición que hará posible elegir la mejor opción para la toma de decisión. Sin embargo, para que esas informaciones lleguen al inversionista es necesario que ocurra una mediación.

En el ámbito de la Ciencias de la Información la mediación es fundamental, ya que analiza un conjunto que incluye toda acción que se puede realizar por profesionales de la información en el proceso de busca y satisfacción de las necesidades informativas de un determinado público (Almeida Júnior, 2008, p. 46):

Mediación de la información es toda acción de interferencia - realizada por profesionales de la información- directa o indirectamente, consciente o inconsciente, singular o plural, individual o colectiva; que promueve la apropiación de información que satisfaga, total o parcialmente, una necesidad de información.

Así, es posible decir que los profesionales involucrados en el proceso de diseminación de informaciones relevantes sobre las empresas de capital abierto pueden ser considerados profesionales de la información. La mediación, en este caso, ocurre a través de la necesidad de las empresas de capital abierto de crear formas de divulgación que permitan utilizar varias medidas, aumentando el número de inversores con acceso a la información. Por lo tanto, es esencial que la mediación entre la empresa y los inversores tenga un carácter efectivo, ya que es a través de esta metodología que la empresa alcanza un nivel satisfactorio de transparencia, característica fundamental de un buen gobierno corporativo.

\subsection{Actuación del Área de Relaciones con los Inversores como mediadora de la información}

A mediados de los años 90, las empresas brasileñas comenzaron un movimiento en pro de la divulgación voluntaria de informaciones al mercado. Tales informaciones permitieron a los inversores y analistas obtener un nivel de conocimiento, cada vez mayor, sobre las empresas, hecho que hizo que las inversiones aumentaran. Sin embargo, fue necesario establecer un conjunto de profesionales capaces de realizar la compilación de informaciones pertinentes $y$, sobre todo, realizar la mediación de esas informaciones en el mercado. Entonces, surgieron los primeros equipos de trabajo centrados en la relación con los inversores en Brasil.
Las áreas de relación con los inversores, conocidas como RI, se centran en la relación efectiva entre la empresa y sus inversores o accionistas. Sus esfuerzos se focalizan en la constante expansión de la calidad de las informaciones divulgadas, en la importancia de la adecuada rendición de cuentas, de la responsabilidad en la conducción de los negocios, en la apertura de canales de comunicación y, sobre todo, en la atención a los inversores.

EI RI debe ser capaz de esclarecer solicitudes específicas, toda vez que, con el desarrollo del mercado de capitales brasileño, hubo un aumento del público interesado en obtener informaciones de esta naturaleza. Este público está compuesto principalmente por los inversores, los analistas del mercado y los periodistas. Cada uno busca informaciones diferentes, ya que tienen diferentes necesidades $y$, por tanto, ofrecen un grado de complejidad informacional diferente. Por lo tanto, los profesionales de la información del área de RI deben estar preparados para resolverlas todas, aunque, desde el punto de vista de la gestión de la información, todos tienen la misma necesidad: la información.

\subsection{Medidas utilizadas en el proceso de la mediación de la información}

Todos las medidas utilizadas por la empresa para el suministro de informaciones deben caracterizarse por la transparencia y la calidad de las informaciones presentadas, de manera que agreguen valor a la consulta del público interesado. Generalmente están disponibles los demostrativos financieros, informes trimestrales, comunicados relevantes, informes anuales, noticias sobre los negocios de la empresa, etc.

De acuerdo con los criterios de confidencialidad, las informaciones pertinentes, a fin de que su divulgación sea oportuna, debe ser hechas públicas. Para la ABRASCA (2007)

Cuando de la divulgación del acto o hecho relevante al mercado, las empresas deben garantizar que la divulgación se haga de manera amplia y sin restricciones, de modo que todo mercado tenga acceso a la información.

La elección de los mejores vehículos para la difusión de informaciones se ha convertido en una parte importante del proceso de gobierno corporativo. En consecuencia, se han producido un aumento en la cantidad de herramientas utilizadas por las empresas para llegar a los diversos públicos interesados en obtener más información. Entre los más populares, destaca el área de RI disponible en los sitios web de las empresas. La velocidad de la diseminación a través de la red Internet es un factor determi- 
nante de la popularidad de este medio de comunicación, ya que proporciona todas las informaciones relevantes en un solo entorno. A continuación, se presentan otros medios de difusión, también muy comunes:

- La publicación de comunicados en los periódicos de gran circulación;

- Circulación de comunicados conocidos como "press releases";

- Conferencias telefónicas realizadas periódicamente;

- Realización de reuniones públicas anuales con analistas.

Es imperativo que la compañía de capital abierto defina metas y objetivos claros y eficaces con respecto a la mediación de la información y, especialmente, que disponga de mecanismos y medidas que faciliten la difusión de informaciones pertinentes a todas las partes interesadas, acción que requiere el establecimiento de criterios y la gestión adecuada de los contenidos divulgados. Por eso, debe haber una evaluación continua de las necesidades de los usuarios con el fin de ajustar las medidas utilizadas hacia mejores niveles de transparencia y calidad de la información, que son las premisas de las mejores prácticas de gobierno corporativo.

\subsection{Construcción del conocimiento como resultado de la mediación eficaz}

Durante el proceso de análisis de las mejores opciones en el mercado de capitales, los inversores utilizan del conocimiento que disponen del mercado y de las empresas, lo que abarca las diversas informaciones disponibles y los medios por los cuales buscan las personas que necesitan de este tipo de información. En consecuencia, es posible considerar que un mercado bien informado está en mejores condiciones de definir un valor razonable para una determinada empresa.

El suministro de informaciones significativas sobre la empresa es una parte esencial de la construcción del conocimiento del inversor. A la medida que este último adquiere informaciones sobre los resultados financieros de la empresa sobre sus proyecciones, sus perspectivas, los negocios realizados, etc., de manera clara, transparente y con patrones coherentes con la realidad, es mayor la probabilidad de que dirija sus propios recursos a la compra de acciones de la empresa analizada. Para Tomaselli (2007), el conocimiento de los inversores es modificado durante el proceso de comunicación, creando y transformando las representaciones sociales del grupo, combinando ciencia y el sentido común, hecho que también cambia la práctica.

Independientemente de los factores macroeconómicos, políticos y sociales, los inversores siempre buscarán informaciones específicas sobre las empresas. Esta actitud se debe al hecho de que, mediante la compra de acciones de una empresa, el inversor es ahora uno de los accionistas, con derecho a recibir los dividendos sobre los beneficios de la empresa. Así, es evidente que un inversor quiera proteger su patrimonio, porque es considerado uno más de los propietarios y, por tanto, necesita obtener informaciones sobre la salud de la empresa. Construir conocimientos basándose en la informaciónn es una actitud que tiende a crecer a medida que el mercado de capitales brasileño se desarrolla.

\section{Consideraciones finales}

Con el desarrollo del mercado de capitales brasileño y el cambio en el nivel de demanda de información por parte de los inversores como un medio para protegerse a sí mismos, la tendencia es el crecimiento y la expansión de los instrumentos del gobierno corporativo, considerados como algo absolutamente esencial. En los últimos años este concepto se ha convertido en un imperativo para las empresas de capital abierto, que quieren seguir siendo competitivas en el escenario nacional e internacional.

Sin embargo, el desempeño de las prácticas de gobierno corporativo está fuertemente condicionado por el nivel de comunicación. Una de las condiciones necesarias para el éxito de estos conceptos es que la mediación de las informaciones entre los inversores y el poder de control de la empresa sea lo más eficiente posible, proporcionando seguridad y transparencia a todos los participantes.

El buen desempeño financiero es fundamental, pero se ha convertido en una exigencia mínima. Hoy en día, la cuestión primordial es la adopción de las mejores prácticas de gobierno corporativo y el compromiso con la transparencia, la ética y el respeto a todas las partes que tienen intereses en la empresa.

Las áreas $\mathrm{RI}$ de varias empresas ofrecen un volumen cada vez mayor de informaciones relevantes y de manera voluntaria, y se puede ver que está creciendo también la preocupación con las medidas utilizadas y con los profesionales involucrados en la mediación de la informaciónn. Las empresas brasileñas han empezado a preocuparse no sólo por cumplir con los requisitos tradicionales, sino también las demandas 
de más información, lo que sin duda mejora considerablemente la credibilidad de la empresa ante los inversores.

\section{Referencias}

Almeida Júnior, O. F. (2008). Mediação da informação: ampliando o conceito de disseminação. // Valentim, M. L. P. (Org.). Gestão da informação e do conhecimento no âmbito da Ciência da Informação. São Paulo: Polis: Cultura Acadêmica, 2008. 41-54

Andrade, A. (2004). Governança corporativa: fundamentos, desenvolvimento e tendências. São Paulo: Atlas, 2004.

Chiavenato, I. (2002). Teoria geral da Administração. 6.ed. Rio de Janeiro: Campus, 2002. 2 v.

Choo, C. W. (2006) A empresa do conhecimento: como as organizações usam a informação para criar significado, construir conhecimento e tomar decisões. São Paulo: SENAC, 2006.

IBGC - Instituto Brasileiro de Governança Corporativa (2008). Código das melhores práticas de governança corporativa. http://www.ibgc.org.br/ibConteudo.asp?ID Area $=864 \& \mid D p=3$ (08 dez. 2008).

Davenport, T. H.; Prusak, L. (1998). Ecologia da informação: por que só a tecnologia não basta para o sucesso na era da informação. São Paulo: Futura, 1998.

Dutra, R. G. (2005). Custos: uma abordagem prática. 5.ed. São Paulo: Atlas, 2003.

Fortuna, E. (2005). Mercado financeiro: produtos e serviços. 16.ed. Rio de Janeiro: Qualytimark, 2005.

ABRASCA - Associação Brasileira de Companhias Abertas (2007). Manual ABRASCA de controle e divulgação de informações relevantes. São Paulo: 2007.

Valentim, M. L. P.; Gelinski, J. V. (2006). Gestão do conhecimento corporativo. // Valentim, M. L. P. (Org.). Informação, conhecimento e inteligência organizacional. Marília: FUNDEPE Editora, 2006. 115-131.

Williamson, O. E. (1996). The mechanisms of governance. Oxford: Oxford university Press, 1996. 